\title{
Characterization of breast tissue using energy-dispersive X-ray diffraction computed tomography
}

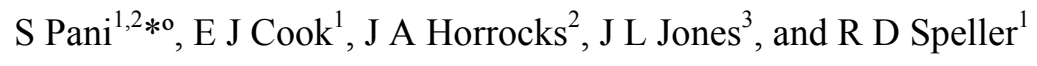

1 Department of Medical Physics and Bioengineering, University College London, UK

2 Clinical Physics, Institute of Cancer, Queen Mary’s School of Medicine and Dentistry/Barts and The London NHS Trust, London, UK

3 Tumour Biology Laboratory, Institute of Cancer, Queen Mary’s School of Medicine and Dentistry/Barts and The London NHS Trust, London, UK

* Corresponding author; e-mail s.pani@surrey.ac.uk

${ }^{\circ}$ Current affiliation: Department of Physics, University of Surrey, Guildford, UK

\begin{abstract}
A method for sample characterization using energy-dispersive X-ray diffraction computed tomography (EDXRDCT) is presented. The procedures for extracting diffraction patterns from the data and the corrections applied are discussed.

The procedures were applied to the characterization of breast tissue samples, $6 \mathrm{~mm}$ in diameter.

Comparison with histological sections of the samples confirmed the possibility of grouping the patterns into five families, corresponding to adipose tissue, fibrosis, poorly differentiated cancer, well differentiated cancer, and benign tumour.
\end{abstract}

\section{Keywords}

Tissue characterization, X-ray diffraction, computed tomography, breast imaging. 


\section{Introduction}

X-ray diffraction (XRD) is typically used for studying the structure of crystalline substances, the inter-atomic and inter-molecular distances determining the presence of sharp peaks characteristic of each material.

However, structures characterized by a certain degree of short-range order, such as amorphous materials or biological tissue, still give rise to diffraction patterns featuring one or more broad peaks.

Diffraction patterns for weakly ordered structures, such as biological tissue, are often expressed as a function of momentum transfer, $\chi=1 / \lambda \sin (\vartheta / 2)$, where $\lambda$ is the $\mathrm{X}$-ray wavelength and $\vartheta$ is the scattering angle. The corresponding inter-atomic or inter-molecular spacing investigated is $d=1 /(2 \chi)$. Hence, different distances can be investigated depending upon the combination of angle and beam energy, X-ray diffraction acting as a probe at the molecular and sub-molecular level. The diffraction properties of amorphous materials, such as biological tissue, are explored in SAXS (Small Angle X-ray Scattering) experiments, based on monochromatic beams and fixed 2-dimensional detectors, or in XRD experiments, based either on monochromatic beams and angular scanning of a point-like detector (angledispersive XRD) or on polychromatic beams and a spectroscopic detector at a fixed angle (energy-dispersive XRD).

SAXS experiments usually explore spacings in the range up to $100 \mathrm{~nm}$, whereas typical XRD experiments deal with spacings the order of a few $\mathrm{nm}$.

Several studies, based both on the SAXS (Lewis et al, 2000; Fernandez et al, 2002; Round et al, 2005; Suhonen et al, 2005; Sidhu et al, 2009) and on the XRD modality (Kidane et al, 1999; Poletti et al, 2002; LeClair et al, 2006; Ryan and Farquharson, 2007; Oliveira et al, 2008), have shown the feasibility of breast tissue characterization. Of particular interest is the possibility of discriminating healthy and neoplastic tissue. This is due to an altered arrangement of collagen in tumour with respect to healthy tissue (Fernandez et al, 2002; Suhonen et al, 2005), to invasion of healthy tissue by tumour causing the residual adipose tissue component to be reduced (Kidane et al, 1999, Ryan and Farquharson, 2007; Oliveira et $a l, 2008)$, and to broadening of diffraction peaks caused by loss of short-range order in neoplastic tissue with respect to healthy tissue.

Several authors have proved the feasibility of breast tissue characterization in the range $0.5-$ $4 \mathrm{~nm}^{-1}$, corresponding to typical molecular spacing of about 1-10 $\AA$. This was done using conventional diffractometers (Poletti et al, 2002; Oliveira et al, 2008), monochromatic synchrotron radiation (Castro et al 2004), or energy-dispersive systems (Kidane et al, 1999; Geraki et al, 2004, LeClair et al, 2006; Ryan and Farquharson, 2007). 
All studies proved that a sharp peak is present in the diffraction profile of adipose tissue for a momentum transfer value $\chi=1.1 \mathrm{~nm}^{-1}$, whereas the diffraction profiles of tumour and of other tissue types have a maximum at about $\chi=1.6 \mathrm{~nm}^{-1}$, due to the predominance of fibroustype tissue, characterized by a large water component. These characteristics were in some cases exploited for momentum transfer-selective diffraction imaging, showing increased contrast compared to conventional imaging. The systems used in these cases were based on monochromatic synchrotron radiation (Harris et al, 2003; Castro et al, 2005; Pani et al, 2007). Characterization of tissue specimens above a few $\mathrm{mm}$ in thickness, less likely to be uniform along the primary beam path, becomes difficult due to the superposition of signals from tissue components placed at different depths. Computed tomography (CT) overcomes this problem, in that it provides a three-dimensional map of the distribution of scatter signatures. X-ray diffraction CT (XRDCT) was initially proposed and treated in detail by Harding et al (1987; 1990). They used two different approaches: in the first one (Harding et al, 1987) images were obtained by tomographic reconstruction of acquired data (sinograms), showing different contrast between details when different momentum transfer values are looked at, whereas in the second one (Harding et al, 1990) the intrinsic volume-selectivity of energy-dispersive Xray diffraction was exploited for a voxel-by-voxel acquisition of diffraction patterns from the volume of interest without the need for a reconstruction.

Both approaches were used in more recent years for a wide range of applications, both in XRD and in SAXS experiments (for instance, Hall et al, 2000; Barroso et al, 2001; Schroer et al, 2006; Bleuet et al, 2008; Cernik et al, 2009). However, the main limitation for a widespread application of the voxel-by-voxel approach lies in the long acquisition times needed to achieve good statistics when information from small voxels is collected. For this reason, this approach is mostly limited to synchrotron sources.

Data based on sinogram reconstruction XRDCT with conventional sources are fewer and limited to highlighting changes in contrast when the information from different momentum transfer values is exploited (Harding et al, 1987; Griffiths et al, 2007).

XRDCT was used for tissue imaging in two studies based on synchrotron radiation (Griffiths et al, 2008) and an on energy-dispersive system with a conventional X-ray tube (Griffiths et $a l$, 2007), respectively. However, these studies only proved the feasibility of tissue discrimination through momentum-transfer selective imaging, featuring increased contrast compared to conventional imaging; the information available within a continuous momentum transfer range, resulting in one case from the possibility of tuning the monochromatic beam energy, and in the other case from the use of a continuous X-ray spectrum, was not fully exploited. 
The novelty of the approach presented in this paper lies in the use of XRDCT for extraction of diffraction patterns of tissue over a range of momentum transfer values. This allows tissue characterization, as opposed to simple discrimination as in the above-quoted works. In particular, identification of benign and malignant lesions, for which few data are available so far (Kidane et al, 1999, Ryan and Farquharson, 2007; Oliveira et al, 2008) was feasible in the present study.

We present procedures for data correction and extraction of diffraction patterns from a set of tomograms, as well as the results on a set of of breast tissue specimens.

\section{Materials and methods}

\subsection{Breast tissue samples}

Eleven samples of normal, benign and malignant breast tissue were derived from surgical tissues excess to diagnosis, following informed patient consent. The samples were snap frozen in liquid nitrogen and stored in the Barts Breast Tissue Bank according to Local Ethics Approval.

They were kept in sealed, thin-walled plastic tubes ( $6 \mathrm{~mm}$ in diameter) with a brass stopper at one end, transported to the laboratory in dry ice and stored at $-35^{\circ}$. During data acquisition they were placed in a brass holder and kept at a temperature between $1{ }^{\circ} \mathrm{C}$ and $2{ }^{\circ} \mathrm{C}$ by means of a thermo-electric module (SH 1.0-95-05, Melcor, USA) equipped with a heat sink. The brass stopper at one end of the plastic tube ensured thermal contact with the holder. This was done in order to prevent tissue degradation while on the other hand keeping it above $0^{\circ}$, as the freezing of water present in tissue would alter the diffraction profile.

Following imaging, the samples were placed in $10 \%$ formal saline for $24-48$ hours, after which they were transacted into three pieces, routinely processed and embedded in paraffin. Sections $(4 \mu \mathrm{m})$ were cut onto glass slides and stained with Haematoxylin and Eosin.

\subsection{The energy-dispersive XRDCT system}

The energy-dispersive XRDCT (EDXRDCT) system was based around the use of a W-anode X-ray tube (MXR-160/0.4-3.0, Comet, Switzerland) with a nominal source size of $3 \mathrm{~mm} \times 3$ $\mathrm{mm}$ and of a high-purity Germanium detector (HPGe) (GLP 16260/13-P-S, EG\&G Ortec, USA) coupled to a standard spectroscopy electronic chain (EG\&G Ortec 855 Dual Spec Amplifier and 921 Spectrum Master) and commercial control software (EG\&G Ortec Maestro). The measured detector energy resolution was $0.4 \mathrm{keV}$ at $59.5 \mathrm{keV}$. During the experiments presented here the tube was operated at $70 \mathrm{kVp}$ and the current was kept constant at $5 \mathrm{~mA}$ for all acquisitions. Rotation and translation were achieved using two stepper motors (RS 440-420; RS Components, Corby, UK), controlled by a drive board (RS 240-7920) and a PC-driven card (NI PCI-6503; National Instruments, USA).

A schematic drawing of the system is shown in figure 1. 
A primary beam collimator $1 \mathrm{~mm}$ in diameter was aligned with the beam axis. The collimator aperture determined the CT slice thickness. The uniformity along the vertical axis of the imaged slices was verified by scanning the samples with a conventional transmission microCT unit (XTek Benchtop; XTek Inc, Tring, UK). In particular, it was checked that no horizontal boundaries between two regions were present in the slices examined, as in this case the reconstructed data could have been affected by partial volume effects.

The sample holder was placed on a set of rotation-translation stages, and a brass conical collimator was placed between the sample and the detector, allowing the collection of photons scattered around $6^{\circ}$. The collimator was $30 \mathrm{~mm}$ long and its aperture was $0.5 \mathrm{~mm}$. The distance between the source and the primary collimator was $400 \mathrm{~mm}$; the distance between the sample and the primary collimator was $15 \mathrm{~mm}$ and the distance between the front of the scatter collimator and the sample was $43 \mathrm{~mm}$.

The angular acceptance of the system for a $6 \mathrm{~mm}$ thick sample, calculated with a similar procedure to that described in Pani et al (2009), had a full-width at half maximum of about $0.7^{\circ}$.

The correlation between the energy $E$ of the scattered photons and the momentum transfer $\chi$ is given by

$$
\chi\left(\mathrm{nm}^{-1}\right)=0.806 \cdot E(\mathrm{keV}) \cdot \sin (\vartheta / 2)
$$

where 0.806 is a conversion factor between energy $(\mathrm{keV})$ and momentum transfer $\left(\mathrm{nm}^{-1}\right)$, and $\vartheta=6^{\circ}$ in this case.

The collimator axis was aligned to the primary beam axis by means of a set of manually controlled rotation and translation stages.

Sinograms were acquired in the "first generation CT" geometry (Swindell and Webb, 1998), with the sample being scanned in front of the primary beam for the acquisition of a profile and being subsequently rotated for the acquisition of the next profile.

A spectrum of scattered radiation was acquired at each scanning step; the sample scanning was synchronized to the acquisition of spectra using a LabView (National Instruments) custom-written program. Data were collected over $360^{\circ}$; for the present study, the linear scanning step was $750 \mu \mathrm{m}$ and the angular scanning step was $1.8^{\circ}$. Each profile consisted of 14 steps. All spectra were acquired for 4 seconds.

The incident beam spectrum was calculated from measurement of the Compton scattered radiation (Maeda et al, 2005). This procedure was adopted in order to avoid pulse pile-up that would occur with a direct measurement. 


\subsection{Image reconstruction}

In scatter CT reconstruction the data are treated as emission CT signals, and the reconstruction is done by simply backprojecting the acquired sinogram.

All data corrections and reconstruction procedures were written in IDL (ITT Visual Information Solutions, USA).

A first approximation to the reconstructed tomogram was obtained by reconstructing the sinogram by means of a filtered backprojection algorithm with a gen-Hamming filter (Herman 1980). A correction for attenuation of X-rays within the sample was applied $a$ posteriori.

\subsubsection{Attenuation correction}

Consider a position $x$ along the direction orthogonal to the beam (i.e. the sample scanning direction).

The numbers of photons of energy $E$ scattered at an angle $\vartheta \pm \mathrm{d} \vartheta$ from a layer of thickness $\mathrm{d} z$ at a depth $z$ (see figure 2) along the beam direction is

$\mathrm{d} N_{s}(x, z, \vartheta, E)=N(x, z, E) \mu_{s}(x, z, \vartheta, E) \mathrm{d} z=N_{0}(E) \cdot \exp \left(-\int_{0}^{z} \mu \mathrm{d} \xi\right) \cdot \mu_{s}(x, z, \vartheta, E) \mathrm{d} z$

where $\mathrm{N}(x, z, E)$ is the number of photons reaching depth $z$ inside the sample, $\mu_{s}$ is the linear differential scatter coefficient in the position $(x, z)$ within the selected sample slice, $N_{0}$ is the number of photons incident on the sample and $\mu=\mu(E)$ is the linear attenuation coefficient.

For pure elastic scattering, $\mu_{s}$ is correlated to the elastically scattered cross section by

$\mu_{s}(\chi)=\frac{N \rho}{M} \frac{\mathrm{d} \sigma_{\mathrm{T}}}{\mathrm{d} \Omega} F_{m}^{2}(\chi)$

where $N$ is Avogadro's number, $M$ is the molecular weight of the material, $\rho$ is the material density, $\left(\mathrm{d} \sigma_{\mathrm{T}} / \mathrm{d} \Omega\right)$ is the Thomson differential cross section and $F_{\mathrm{m}}^{2}$ is the molecular form factor, describing the spatial distribution of electrons responsible for the interference of the scattered waves and hence for the shape of the diffraction pattern.

Scattered photons are attenuated by the underlying layers of tissue. Assuming that the angle $\vartheta$ is small, and hence that the approximation $D \sim D \cos (\vartheta)$ holds, that the sample composition varies slowly along the vertical axis and that only single scatter events occur along the thickness of the sample (assumption acceptable due to the small thicknesses involved), the number of detected photons can be approximated as

$$
d N_{\text {det }}(x, \vartheta, z, E)=\mathrm{d} N_{s}(x, \vartheta, z, E) \cdot \exp \left(-\int_{z}^{L} \mu \mathrm{d} \xi\right)=N_{0}(E) \cdot \exp \left(-\int_{0}^{L} \mu \mathrm{d} \xi\right) \cdot \mu_{s}(x, z, \vartheta, E) \mathrm{d} z
$$

where $L=L(x)$ is the thickness of the sample along the direction parallel to the primary beam in the position $x$.

The total number of detected photons of energy $E$ is hence 


$$
N_{\text {det }}(x, \vartheta, E)=\int_{0}^{L} \mathrm{~d} N_{\text {det }}(x, z, \vartheta, E)=N_{0}(E) \exp \left(-\int_{0}^{L} \mu \mathrm{d} \xi\right) \cdot \int_{0}^{L} \mu_{s}(x, z, \vartheta, E) \mathrm{d} z
$$

from which we have

$$
\int_{0}^{L} \mu_{s}(x, z, \vartheta) \mathrm{d} z=\frac{N_{\operatorname{det}}(x, \vartheta)}{N_{0} \exp \left(-\int_{0}^{L} \mu \mathrm{d} z\right)}
$$

Now a distribution map of $\mu_{s}$ can be obtained with a mathematically correct reconstruction procedure because its line integrals collected across $360^{\circ}$ are known (Kak and Slaney 2001). In the case presented here, the line integrals can be assumed to cover the whole thickness of the sample because the sample thickness is entirely contained within the scatter volume length: using trigonometry, the latter was calculated to be $30 \mathrm{~mm}$. It must be noted that the use of this geometrical approach in conical collimation is an approximation because photons actually cross different portions of the sample. This causes a blur in the margins of the regions of interest.

The quantity $\exp \left(-\int_{0}^{L} \mu \mathrm{d} z\right)$ can be obtained if a map of the linear attenuation coefficients is available.

The attenuation coefficient map was obtained for all samples based on two diffraction tomograms obtained at $1.1 \mathrm{~nm}^{-1}$ and at $1.6 \mathrm{~nm}^{-1}$, respectively. As shown in the quoted studies related to this momentum transfer range, the diffraction pattern from adipose tissue has a peak at $1.1 \mathrm{~nm}^{-1}$, whereas other tissue types give rise to a higher signal than adipose tissue at 1.6 $\mathrm{nm}^{-1}$. Hence, by setting two appropriate thresholds at the two momentum transfer values it is possible to discriminate the adipose tissue distribution from that of other tissue types. Based on this fact, a distribution of attenuation coefficients was generated for all spectrum channels, assigning to the adipose tissue area the attenuation coefficient of adipose tissue for the corresponding energy as calculated from the XMuDat software (Nowotny, 1998) and to the area corresponding to other tissue types the attenuation coefficient of water as calculated using the same software. Water was considered an acceptable approximation, as the maximum difference in the energy range of interest between the attenuation coefficients of water and those of fibrous tissue and of infiltrating ductal carcinoma (IDC), as published by Johns and Yaffe (1987), was found to be $1 \%$ for fibrous tissue and $4 \%$ for IDC, leading to maximum differences in attenuation along a $6 \mathrm{~mm}$ path (maximum length to be crossed by $\mathrm{X}$ rays) of $0.6 \%$ and of $3.5 \%$, respectively.

The above described procedure was considered to be more effective than the collection of separate transmission tomograms and the extraction of attenuation coefficients from them, as 
the latter procedure would have required sample repositioning and hence the use of image registration algorithms.

In the adopted method, the segmented transmission $\mathrm{CT}$ images are intrinsically co-registered to the diffraction ones.

\subsubsection{Depth response correction}

The depth response of the system, related to its geometrical characteristics, was obtained by scanning a thin scatterer along the direction parallel to the primary beam, measuring the total scattered intensity and by fitting the experimental points with a third-order polynomial. The result is shown in figure 3.

A simulation was carried out to prove that the effect of depth response on the reconstructed image is negligible. A uniform object was simulated within a matrix the same size as the reconstructed images. The object was rotated; at each rotation step each column of the array was multiplied by the depth response of the system, and the resulting matrix was projected into a line of the sinogram. The sinogram was reconstructed using filtered backprojection and the resulting image was compared to the image obtained by reconstructing a sinogram obtained without depth response correction. Figure 4 shows a profile across the central row of the reconstructed images, proving that the effect of depth response after filtration and backprojection is negligible.

\subsubsection{Data reconstruction}

EDXRDCT data were processed according to the following procedure, based on an IDL Graphical User Interface (GUI) for data entering and display:

- Each acquired spectrum is corrected by background subtraction and normalization by incident beam spectrum;

- A sinogram is built for each momentum transfer value. The sinogram for the $k$-th momentum transfer value is obtained as an $(m \times n)$ array, where $m$ is the number of steps for each profile and $n$ is the number of angular positions. The $(i, j)$ pixel of the sinogram for the $k$ th momentum transfer value corresponds to the counts in the $k$-th bin of the $s$-th spectrum acquired, where $s=(i+j \times m)$, the index $i$ running from 0 to $m-1$ and the index $j$ running from 0 to $n-1$.

- Attenuation correction is applied by observing that $N_{\text {det }}(x, \vartheta, E)$ is the sinogram component acquired at a position $x$ for the energy component chosen and the term $N_{0}(E)$ is the incident spectrum intensity for the same component. The term $N_{\text {det }}(x, \vartheta, E) / N_{0}(E)$ is directly proportional to the term obtained by dividing the measured scattered spectrum by the incident spectrum; the proportionality only depends on the geometry and is constant across the whole momentum transfer range. Hence, dividing the corrected sinograms by the term $\exp \left(-\int_{0}^{L} \mu d z\right)$ 
as calculated from the segmented images gives a quantity that is directly proportional to the line integral of $\mu_{\mathrm{s}}$.

- A tomogram is reconstructed for each spectrum channel using the procedure described in Section 2.3.

- The user selects a region of interest from a tomogram at any of the momentum transfer values; the program automatically calculates the average intensity in the same ROI for all tomograms, each one corresponding to a different momentum transfer value. The diffraction pattern for that region is the plot of the average intensity vs momentum transfer.

\section{Results}

\subsection{Images and histopathology sections}

Figure 5 shows EDXRDCT images of a two-component tissue at two different momentum transfer values, together with the corresponding histological section of the tissue. The images were obtained by averaging 11 tomograms of the same CT slice, each one corresponding to a different energy/momentum transfer, centred around the desired momentum transfer value.

The two main regions of interest are marked on all images, region A corresponding to adipose tissue and region $\mathrm{B}$ corresponding to fibrosis. The fact that the fibrosis region is not visible in the images at $1.1 \mathrm{~nm}^{-1}$ and the adipose region is poorly visible at $1.6 \mathrm{~nm}^{-1}$ depends upon the large difference between their diffraction patterns at the two momentum transfer values (see figures 7 and 9).

Figure 6 shows EDXRDCT images of another two-component sample at the same two momentum transfer values as above, together with the corresponding histological section of the tissue.

In this case, region $\mathrm{A}$ corresponds to adipose tissue and region $\mathrm{B}$ corresponds to well differentiated cancer. In this case, the two regions are not simultaneously visualized in either image due to the large difference in the scattered intensity from these tissue types. On the other hand, fibrous tissue and adipose tissue (figure 5) are simultaneously visualized at 1.6 $\mathrm{nm}^{-1}$ due to the smaller difference in their diffraction patterns for that momentum transfer value.

\subsection{EDXRDCT patterns}

A total of 23 EDXRDCT patterns was obtained as described in Section 2.3.3 from all regions of interest identified in the images. Figure 7 shows these patterns, grouped by tissue type. The close agreement of all curves within each group confirms that the contribution of partial volume effects are limited even with a relatively thick CT slice (approximately $1 \mathrm{~mm}$ ) and that the patterns measured are true diffraction profiles of tissue. Partial volume effects would cause continuous variations in the profiles and it would not be possible to group them into separate families. 
The group of curves for adipose tissue shows a larger variation in intensity than the other groups. This is related to larger variations in density, resulting from the higher compressibility of adipose tissue compared to fibrous-type tissue (Stavros, 2004).

The statistical significance of the grouping of the diffraction patterns in five families was proved by Principal Component Analysis (PCA) using a standard multivariate analysis package (Unscrambler; CAMO, Norway). The scores plot for the two first principal components is shown in Figure 8. The residual variance after one principal component was $98 \%$. Hence, one principal component is sufficient to describe $98 \%$ of the variations from one set of samples to another: this is reflected in the fact that samples of the same tissue type have a close vertical alignment in the scores plot. Also in this case, the largest data scatter is found for the adipose tissue set, reflecting the intensity variations discussed above.

The average curves of all groups are shown together in figure 9. The data have been smoothed using Savitzky-Golay filtering (Savitzky and Golay, 1964) for easier comparison. Although Savitzky-Golay filtering is recommended for noisy data with sharp peaks, not present in diffraction patterns from biological tissue, this algorithm was used for uniformity with the typical procedures for X-ray diffraction data processing. It must be noted that two different patterns correspond to cancer: one corresponds to poorly differentiated infiltrating ductal carcinoma (IDC) and one to well differentiated IDC. Figure 10 shows a comparison between two histological sections of poorly differentiated IDC and well differentiated IDC, respectively. Their different tissue structure is reflected in the difference in the XRD patterns.

\section{Discussion and conclusions}

Although several authors (Kidane et al, 1999; Castro et al, 2004; Geraki et al 2004; LeClair et al, 2006; Ryan and Farquharson, 2007; Oliveira et al, 2008) have proved that a different diffraction signal is achieved from normal and neoplastic breast tissue in the momentum transfer range $0.5-4 \mathrm{~nm}^{-1}$, there are few data about identification of different lesion types. In this study, the diffraction patterns of different lesions have been investigated using EDXRDCT.

The main advantage of CT compared to planar diffraction analysis is the possibility of separating the contributions from details placed at different depths within the sample, thus allowing more reliable characterisation. This appears particularly suitable for the characterisation of larger samples, where the overlap of different tissue components is unavoidable.

The technique proposed for EDXRDCT, consisting of extracting XRD patterns from energydispersive tomograms, can be used for material characterization in other fields where different components may be overlapping in a sample.

In the present study, no correction for Compton scatter has been applied. This should in principle be needed because Compton scatter becomes important compared to coherent scatter 
in biological tissue above about 20-30 keV (Nowotny, 1998). However, the goal of this study was to prove the feasibility of tissue characterisation in terms of its histological characteristics, for which the procedure adopted appears effective because Compton scatter forms a non-structured background. Corrections for Compton scatter would be needed for larger samples and in studies aimed at characterising the molecular and sub-molecular structure of samples, as the Compton background may alter the relative height of peaks.

For similar reasons, polarisation corrections, likely to affect the highest energy components of the spectrum were not applied. Moreover, because of the low scatter angle involved for the present experiment, it was found that even the largest absolute value of polarization measured by Olsen et al (1978) would only bring a maximum correction of less than $0.5 \%$ to the highest energy components.

It can be seen from the EDXRDCT patterns in figure 9 that the spectral components carrying most information are centred around $1.1 \mathrm{~nm}^{-1}$ (adipose tissue peak) and $1.6 \mathrm{~nm}^{-1}$ (fibrous tissue peak). Comparison of the scattered intensities at both these values of momentum transfer allows discrimination of any of the tissue types studied, whereas the measured intensity at $1.1 \mathrm{~nm}^{-1}$ alone would not allow the discrimination of poorly differentiated cancer from well differentiated cancer and that at $1.6 \mathrm{~nm}^{-1}$ alone does not allow the discrimination of benign tumour from well differentiated cancer. However, the use of the full pattern, rather than of selected momentum transfer windows, would be more reliable in the case of statistically poor data: in this way, fluctuations affecting a single component of the spectrum would not prevent classification.

For instance, in the case of a comparison between benign tumor and well-differentiated cancer, even if noise caused the intensities of the two patterns at $1.1 \mathrm{~nm}^{-1}$ and $1.6 \mathrm{~nm}^{-1}$ to overlap, the averaged intensity between 1.5 and $2.8 \mathrm{~nm}^{-1}$ could still be lower, with statistical significance, for well-differentiated cancer than for benign tumour.

The predominance of the two above mentioned momentum transfer components in describing variations between different tissue types has been observed by several authors (Kidane et al, 1999; Ryan and Farquharson, 2007; Oliveira et al, 2008 to include those who have addressed different type of tumours). A quantitative comparison is difficult due to the likely differences in the experimental set-ups used (for instance, different angular resolutions are likely to affect the peak width and height), to the exiguity and sparseness of data available for non-adipose tissue types and to lack of information on some experimental set-ups. A qualitative comparison shows a good agreement of the present data, in particular for what concerns the changes in the $1.1 \mathrm{~nm}^{-1}$ component from benign tumour to cancer, with the data published by Ryan and Farquharson (2007). This is not observed in the data published by Oliveira et al (2008); however, the information provided by these authors regarding sample preparation or data scaling for different samples is not exhaustive, and differences may result from 
contributions to the diffraction patterns of different tissue types. The shape of the curve for fibrosis also matches that obtained by Ryan and Farquharson (2007) for fibrocystic changes, but the relative heights of these curves compared to that of adipose tissue do not match. Also in this case, more information should be needed on the experimental set-up.

Moreover, it must be pointed out that this work was not aimed at providing an absolute measurement of linear differential scatter coefficients, but rather at proving the feasibility of on-line tissue identification using XRDCT with a view to automatic classification. For this reason, corrections for Compton scatter were not applied.

The differences in the diffraction patterns for benign tumour, well and poorly differentiated cancer are related to the different mechanisms of growth for the different lesions. The low level of the adipose component in the benign tumour pattern, also observed by Ryan and Farquharson (2007), is related to the larger fibrous component associated with the development of the benign tumour. On the other hand, cancer can still be associated with the presence of fat since cancerous cells infiltrate pre-existing adipose tissue, and may not be associated with a fibrotic reaction.

Poorly differentiated cancers are frequently highly cellular with little fibrotic stroma or adipose tissue. The fibrous structure has been largely lost, as can be seen in Figure 10a, and the fibrous peak in the diffraction pattern is therefore depressed.

The main limitation of the EDXRDCT system described, based upon the first generation CT geometry, is the long acquisition times needed. There are several options for reducing this limitation. One is the use of faster detectors, such as Cadmium Zinc Telluride (CZT). Although CZT is characterized by poorer energy resolution than HPGe, the presence of relatively broad peaks in the diffraction patterns of biological tissue suggests that the high resolution of a HPGe detector is not required, and that the resolution of CZT (typically up to 2-4 keV FWHM in the energy range of interest - Dedek et al, 2007; Veale et al, 2007) is sufficient. Moreover, the contribution of energy resolution to the broadening diffraction patterns obtained with typical energy-dispersive systems is smaller than that of angular resolution (Pani et al, 2009).

Another option could be the use of detector arrays coupled to appropriately designed multihole scatter collimators, matching the beam divergence, allowing the simultaneous acquisition of one profile.

Further work will be done on these aspects, on the improvement of spatial resolution (by using finer collimation and a finer sampling step) and on the improvement of sample positioning, thus obtaining a more accurate comparison of histopathology and EDXRDCT.

The technique presented has high potential for the diagnosis of breast cancer. The possibility of discriminating cancer, fibrosis and benign tumour, as well as the feasibility of cancer staging (discrimination between poorly differentiated and well differentiated cancer), appears 
particularly promising, in that neither of these tasks is feasible in conventional imaging. Further studies will be conducted on systems with improved spatial and momentum transfer resolution, and with larger data-sets, to assess the feasibility of characterizing more lesion types, including pre-cancerous lesions with varying prognosis, and to identify any other momentum transfer components carrying information for this purpose.

Although an in vivo use of the technique is not immediately feasible, XRDCT could be used as a biopsy aid for analysing ex-vivo tissue samples. The technique could be coupled to segmentation algorithms and statistical methods, such as multivariate analysis, for automatic identification and classification of lesions. The procedure would allow fast characterization of specimens both in a histopathology laboratory and in the operating theatre, and could ease the workload on health service staff.

\section{Acknowledgments}

Silvia Pani was supported, during part of this work, by a Marie Curie Intra-European Fellowship (MEIF-CT-2004-007206) within the $6^{\text {th }}$ European Framework Programme. 


\section{References}

Barroso, R.C., Lopes, R.T., Gonçalves, O.D., de Assis, J.T. 2001. X-ray diffraction microtomography using synchrotron radiation. Nucl. Instr. Meth. A 471, 75-79

Bleuet, P., Welcomme, E., Dooryhee, E., Susini, J., Hodeau, J.L. Walter, P. 2008. Probing the structure of heterogeneous diluted materials by diffraction tomography. Nature Materials 7, 468-472

Castro, C.R.F., Barroso, R.C., Anjos, M.J., Lopes, R.T., Braz, D. 2004. Coherent scattering characteristics of normal and neoplastic breast tissue. Rad. Phys. Chem. 71 649-651

Castro, C.R.F., Barroso, R.C., de Oliveira, L.F., Lopes, R.T. 2005. Coherent scattering X-ray imaging at the Brazilian National Synchrotron Laboratory: Preliminary breast images. Nucl. Instr. Meth. A 548 116-122

Cernik, R.J., Khor, K.H., Hansson C. 2008. X-ray colour imaging. J. R. Soc. Interface 5, 477481

Dedek, N.P., Speller, R.D., Spendley, P., Horrocks J.A. 2007. Performance evaluation of 98 CZT sensors for their use in gamma-ray imaging. Proceedings IEEE-NSS 3 2386-2391

Fernandez, M., Keyrilainen, J., Serimaa, R., Torkkeli, M., Karjalainen-Lindsberg, M. L., Tenhunen, M., Thomlinson, W., Urban, V., Suortti, P. 2002. Small-angle x-ray scattering studies of human breast tissue samples Phys. Med. Biol. 47 577-92

Geraki, K., Farquharson, M.J., Bradley, D.A. 2004. X-ray fluorescence and energy dispersive $\mathrm{x}$-ray diffraction for the quantification of elemental concentrations in breast tissue. Phys. Med. Biol. 49 99-110.

Griffiths, J.A., Royle, G.J., Hanby, A.M., Horrocks, J.A., Bohndiek, S.E., Speller, R.D. 2007. Correlation of energy dispersive diffraction signatures and microCT of small breast tissue samples with histological analysis. Phys. Med. Biol. 52 6151-6164

Griffiths, J.A., Royle, G.J., Horrocks, J.A., Hanby, A.M., Pani, S., Speller, R.D. 2008. Angular dispersive diffraction microCT of small breast tissue samples. Rad. Phys. Chem. 77, 373-380

Hall, C., Colston, S.L., Jupe, A.C., Jacques, S.D.M., Livingston, R., Ramadan, A.O.A., Made, W.A., Barnes, P. 2000. Non destructive tomographic energy-dispersive diffraction imaging of the interior of bulk concrete. Cement and Concrete Research 30, 491-495

Harding, G., Kosanetzky, J., Neitzel, U. 1987. X-ray diffraction computed tomography. Med. Phys. 14, 515-525

Harding, G., Newton, M., Kosanetzky, J. 1990. Energy-dispersive X-ray diffraction computed tomography. Phys. Med. Biol. 35, 33-41

Harris, E.J., Royle, G.J., Speller, R.D., Griffiths, J.A., Kidane, G., Hanby, A.M. 2003. Evaluation of a novel low light level (L3 vision) CCD technology for application to diffraction enhanced breast imaging. Nucl. Instr. Meth. A 513, 27-31

Herman, G.T. 1980. Image reconstruction from projections, Academic Press, New York.

Johns, P.C. and Yaffe, M.J. 1987. X-ray characterization of normal and neoplastic breast tissue. Phys. Med. Biol. 32 675-695

Kak, A.C. and Slaney, M. 2001. Principles of Computerized Tomographic Imaging, Philadelphia: Society for Industrial and Applied Mathematics.

Kidane, G., Speller, R.D., Royle, G.J., Hanby, A.M. 1999. X-ray scatter signatures for normal and neoplastic breast tissues. Phys. Med. Biol. 44 1791-1802

LeClair, R.J., Boileau, M.M., Wang, Y. 2006 A semianalytic model to extract differential linear scattering coefficients from energy dispersive x-ray diffraction measurements. Med. Phys. 33, 959-967

Lewis, R.A., Rogers, K.D, Hall, C.J, Towns-Andrews, E., Slawson, S., Evans, A., Pinder, S.E., Ellis, I.O., Boggis, C.R.M., Hufton, A.P., Dance, D.R. 2000. Breast cancer diagnosis using scattered X-rays. J. Synchrotron Rad. 7, 348-352

Maeda. K., Matsumoto, M., Taniguchi, A. 2005. Compton-scattering measurement of diagnostic x-ray spectrum using high-resolution Schottky CdTe detector. Med. Phys. 32 15421547

Nowotny, R. 1998. XmuDat: Photon attenuation data on PC. IAEA report IAEA-NDS 195, Vienna 
Oliveira, O. R., Conceiçao, A.L.C., Cunha, D.M., Poletti, M.E., Pelà, C.A 2008 Identification of neoplasias of breast tissue using a powder diffractometer. J. Radiat. Res. 49, 527-532

Olsen, J.O., Buras, B., Jensen, T. 1978. Influence of polarization of the incident beam on integrated intensities in X-ray energy-dispersive diffractometry. Acta Crystallographica A 34, 84-87

Pani, S., Royle, G., Speller, R., Castoldi, A., Galimberti, A., Guazzoni, C. 2007. Use of a novel Controlled Drift Detector for Diffraction Enhanced Breast Imaging. Nucl. Instrum. Meth. A 573 133-136.

Pani, S., Cook, E.J., Horrocks, J.A., George, L., Speller, R.D. 2009. Modeling an energydispersive X-ray diffraction system for drug detection. IEEE Trans. Nucl. Sci.56, 1238-1241.

Poletti, M.E., Gonçalves, O.D., Mazzaro, I. 2002. X-ray scattering from human breast tissues and breast-equivalent materials. Phys. Med. Biol. 47 47-63

Round, A.R., Wilkinson, S.J., Hall, C.J., Rogers, K.D., Glatter, O., Wess, T., Ellis, I.O. 2005. A preliminary study of breast cancer diagnosis using laboratory based small angle x-ray scattering. Phys. Med. Biol. 50 4159-4168

Ryan, E.A. and Farquharson, M.J. 2007. Breast tissue classification using x-ray scattering measurements and multivariate data analysis. Phys. Med. Biol. 52 6679-6696

Savitzky, A. and Golay, M.J.E. 1964 Smoothing and Differentiation of Data by Simplified Least Squares Procedures. Anal. Chem. 36 1627-1639

Schroer, C.G., Kuhlmann, M., Roth, S.V., Gehrke, R., Stribeck, N., Almendarez-Camarillo, A., Lengeler, B. 2006. Mapping the local nanostructure inside a specimen by tomographic small-angle x-ray scattering. Appl. Phys. Lett. 88, 164102

Sidhu, S., Siu, K.W., Falzon,G., Hart, S.A., Fox, J.G., Lewis, R.A. 2009. Mapping structural changes in breast disease using X-ray scattering. Med. Phys. 36, 3211-3217

Stavros, A.T. 2004. Breast Ultrasound, p. 958. Lippincott Williams and Wilkins, Philadelphia.

Suhonen, H., Fernandez, M., Serimaa, R., Suortti, P. 2005. Simulation of small-angle X-ray scattering from collagen fibrils and comparison with experimental patterns. Phys. Med. Biol. $505401-5416$

Swindell, W. and Webb, S. 1998. X-ray transmission computed tomography. In "The physics of medical imaging", S. Webb ed. London: IOP Publishing

Veale, M.C., Sellin, P.J., Lohstroh, A., Davies, A.W., Parkin, J., Seller, P. 2007 X-ray spectroscopy and charge properties of CdZnTe by the vertical Bridgman method. Nucl. Instr. Meth. A 576 90-94 


\section{Figure captions}

Figure 1. Schematic drawing of the EDXRDCT system (not to scale).

Figure 2. Schematic diagram of the frame of reference used for calculation of attenuation correction.

Figure 3. Depth response of the EDXRDCT system.

Figure 4. Effect of depth response on CT reconstruction. The plots show the profile along the central line of a CT reconstruction of a uniform sample. The non uniform depth response adopted is shown in Figure 3.

Figure 5. Images of a breast tissue sample. a) Reconstructed diffraction tomogram at $1.1 \mathrm{~nm}^{-}$ ; b) Reconstructed diffraction tomogram at $1.6 \mathrm{~nm}^{-1}$; c) histopathology. The dashed lines represent the contours of the different regions, not visualized simultaneously in Figure 5a due to the large contrast between them at $1.1 \mathrm{~nm}^{-1}$. The region marked " $\mathrm{A}$ " is adipose tissue; the region marked " $\mathrm{B}$ " is fibrosis.

Figure 6. Images of a breast tissue sample. a) Reconstructed diffraction tomogram at $1.1 \mathrm{~nm}^{-}$ ${ }^{1}$; b) Reconstructed diffraction tomogram at $1.6 \mathrm{~nm}^{-1}$; c) histopathology. The dashed lines represent the contours of the different regions, not visualized simultaneously in Figures $6 \mathrm{a}$ and $6 \mathrm{~b}$ due to the large contrast between them. The region marked " $\mathrm{A}$ " is adipose tissue; the region marked " $\mathrm{B}$ " is tumour.

Figure 7. Comparison of the diffraction patterns obtained for the different tissue types, showing the close agreement within each group. (a) adipose tissue; (b) fibrosis; (c) poorly differentiated cancer; (d) well differentiated cancer; (e) benign tumour. In each graph, different colours correspond to the diffraction patterns obtained from different samples (or different regions within a sample) with the same histological classification. There is no relationship between the colours used in different graphs.

Figure 8. Principal Component Analysis scores plot for the five tissue groups identified.

Figure 9. Comparison between EDXRDCT patterns of all tissue types identified. The two most significant momentum transfer values for tissue identification are marked. 
Figure 10. Comparison between the histological sections of poorly differentiated IDC (a) and well differentiated IDC (b). 